\title{
Decreased Intracellular pH Induced by Cariporide Differentially Contributes to Human Umbilical Cord-Derived Mesenchymal Stem Cells Differentiation
}

\author{
Wei Gao a,b,c Hairui Zhang ${ }^{a, c}$ Guoqiang Chang ${ }^{a}$ Zhenqing Xie ${ }^{a} H^{2}$ hany Wang ${ }^{a}$ \\ Li Ma $^{a}$ Zhongchao Han ${ }^{\mathrm{a}}$ Qinghua Lia Tianxiang Panga \\ aState key Laboratory of Experimental Hematology, Institute of Hematology and Hospital of \\ Blood Diseases, Chinese Academy of Medical Sciences and Peking Union Medical College, Tianjin, \\ bDepartment of pathophysiology, Weifang Medical College, Weifang, Shandong, China; \\ cJoint first authors: these two authors contributed equally to this work
}

\section{Key Words}

$\mathrm{Na}+/ \mathrm{H}+$ exchanger 1 (NHE1) • Mesenchymal stem cells • Intracellular $\mathrm{pH}\left(\mathrm{pH}_{\mathrm{i}}\right) \cdot$ Osteogenic differentiation $•$ Adipogenic differentiation $\bullet \beta$-catenin

\begin{abstract}
Background/Aims: $\mathrm{Na}+/ \mathrm{H}+$ exchanger 1 (NHE1) is an important regulator of intracellular $\mathrm{pH}\left(\mathrm{pH}_{\mathrm{i}}\right)$. High $\mathrm{pH}_{\mathrm{i}}$ is required for cell proliferation and differentiation. Our previous study has proven that the $\mathrm{pH}_{\mathrm{i}}$ of mesenchymal stem cells is higher than that of normal differentiated cells and similar to tumor cells. NHE1 is highly expressed in both mesenchymal stem cells and tumor cells. Targeted inhibition of NHE1 could induce differentiation of K562 leukemia cells. In the present paper we explored whether inhibition of NHE1 could induce differentiation of mesenchymal stem cells. Methods: MSCs were obtained from human umbilical cord and both the surface phenotype and functional characteristics were analyzed. Selective NHE1 inhibitor cariporide was used to treat human umbilical cord-derived mesenchymal stem cells (hUC-MSCs). The $\mathrm{pH}_{\mathrm{i}}$ and the differentiation of hUC-MSCs were compared upon cariporide treatment. The putative signaling pathway involved was also explored. Results: The $\mathrm{pH}_{\mathrm{i}}$ of hUC-MSCs was decreased upon cariporide treatment. Cariporide up-regulated the osteogenic differentiation of hUC-MSCs while the adipogenic differentiation was not affected. For osteogenic differentiation, $\beta$-catenin expression was up-regulated upon cariporide treatment. Conclusion: Decreased $\mathrm{pH}_{\mathrm{i}}$ induced by cariporide differentially contributes to hUC-MSCs differentiation.
\end{abstract}




\section{Introduction}

It is well known that the intracellular $\mathrm{pH}\left(\mathrm{pH}_{\mathrm{i}}\right)$ of terminally differentiated cells is lower than the relatively more "stem" cells such as tumor cells and/or stem cells $[1,2]$. Higher $\mathrm{pH}_{\mathrm{i}}$ is important to keep the stemness of tumor cells and/or stem cells. The $\mathrm{pH}_{\mathrm{i}}$ is regulated by the transport system located in the plasma membrane, of which NHE1 is the most important one. NHE1 is a member of the $\mathrm{Na}+\mathrm{H}+$ exchanger family and regulates $\mathrm{pH}_{\mathrm{i}}$ by catalyzing the extrusion of intracellular proton ions in exchange for extracellular sodium ions [3-6]. Increased $\mathrm{pH}_{\mathrm{i}}$ caused by the NHE1 has been proved to provide extremely crucial signal for cell proliferation and differentiation, especially in tumor cells [7]. Our previous study has shown that targeted inhibition of NHE1 induced differentiation of K562 leukemia cells [8].

Mesenchymal stem cells (MSCs), also known as mesenchymal stromal stem cells and mesenchymal stromal cells, are a heterogeneous multipotent population of stromal cells with fibroblast-like morphology and proliferate in vitro as plastic-adherent cells. They also possess the properties to form colonies in vitro and differentiate into osteoblasts, chondrocytes and adipocytes [9-12]. In addition to the bone marrow stroma, MSCs have been found in other tissues, such as adipose, umbilical cord blood, umbilical cord (UC), amniotic membrane, gingiva, thymus, placenta, synovium and fetal blood and liver [13-21]. Human umbilical cordderived mesenchymal stem cells (hUC-MSCs) are isolated from discarded extra-embryonic tissue after birth and become a promising source of mesenchymal stem cells.

In this study, we chose hUC-MSCs which have many advantages over other MSCs as the subject. Cariporide, which is a high selective and low cytotoxic NHE1 inhibitor [22], was used to decrease the $\mathrm{pH}_{\mathrm{i}}$. This study was aimed to explore the effect of decreased $\mathrm{pH}_{\mathrm{i}}$ on the differentiation of hUC-MSCs and to explore the putative mechanism involved.

\section{Materials and Methods}

Preparation of hUC-MSCS

Human UC were collected from healthy full-term pregnant donors with written consent and approval by the institutional review board of the Chinese Academy of Medical Sciences and Peking Union Medical College (Tianjin, China). hUC-MSCs were isolated and expanded as described previously [23]. hUC-MSCs were cultured in complete culture medium containing Dulbecco's minimum essential medium (DMEM)/ F12 (1:1) (Gibco; Grand Island, NY, USA) supplemented with 10\% fetal bovine serum (FBS; HyClone, Logan, UT, USA), 100 U/mL penicillin-streptomycin (Sigma; St. Louis, MO, USA), 1\% glutamine (Sigma) and $10 \mathrm{ng} /$ $\mathrm{mL}$ epidermal growth factor (EGF; Sigma). The hUC-MSCs were incubated in a $5 \% \mathrm{CO}_{2}$ incubator at $37{ }^{\circ} \mathrm{C}$, and cells at passage (P) 4-6 were used for experiments unless otherwise stated. The hUC-MSCs used in each independent comparison assay were derived from the same UC in order to reduce experimental variation.

\section{Characteristics of hUC-MSCS}

The phenotype of hUC-MSCs were analyzed by flowcytometry according to the criteria by ISCT [10]. The following antibodies were used for fluorescence-activated cell sorting (FACS) analysis: phycoerythrin (PE)-conjugated antibodies against CD11b, CD45, CD73, CD90, CD105 and HLA-DR, while fluorescein isothiocyanate (FITC)-conjugated antibodies against CD19 and CD34. All antibodies were purchased from Becton Dickinson (San Diego, CA, USA).

Cytotoxicity analysis

MTT assay was performed to detect the cytotoxicity of cariporide to hUC-MSCs. Briefly, cells were seeded into 96-well culture plates at a density of $5 \times 104$ cells $/ \mathrm{ml}$. Cariporide was added in medium with a final volume of $200 \mu \mathrm{l}$ per well at gradient concentrations. After drug treatment for $72 \mathrm{~h}$, medium was replaced with an equal volume of fresh medium containing $0.5 \mathrm{mg} / \mathrm{ml}$ MTT. The culture plates were incubated for $4 \mathrm{~h}$ at $37^{\circ} \mathrm{C}$. Then, the medium was removed and $100 \mu \mathrm{l}$ DMSO was added and incubated for $10 \mathrm{~min}$ at room temperature. The cytotoxic effects of drugs were determined according to the OD values using a microplate reader at absorption wavelength of $490 \mathrm{~nm}$. 
Table 1. The sequence of primers designed for Real-time PCR

\begin{tabular}{|c|c|}
\hline Gene & Primer sequence $\left(5^{\prime}-3^{\prime}\right)$ \\
\hline \multirow[t]{2}{*}{ CEBPD } & Forward: GAAGTTGGTGGAGCTGTCGG \\
\hline & Reverse: TGAGGTATGGGTCGTTGCTGA \\
\hline \multirow[t]{2}{*}{ LPL } & Forward: TGGATGGAGGAGGAGTTT \\
\hline & Reverse: GGATCGAGGCCAGTAATT \\
\hline \multirow[t]{2}{*}{ BMP } & Forward: GACGAGGTCCTGAGCGAGTT \\
\hline & Reverse: ACCTGAGTGCCTGCGATACA \\
\hline \multirow[t]{2}{*}{ Osteocalcin } & Forward: CAGGCGCTACCTGTATCAATG \\
\hline & Reverse: CTCCTGAAAGCCGATGTGGT \\
\hline \multirow[t]{2}{*}{$\beta$-Catenin } & Forward: GAGCCTGCCATCTGTGCTCT \\
\hline & Reverse: ACGCAAAGGTGCATGATTTG \\
\hline \multirow[t]{2}{*}{ GAPDH } & Forward: GAAGGTGAAGGTCGGAGTC \\
\hline & Reverse: GAAGATGGTGATGGGATTTC \\
\hline
\end{tabular}

Evaluation of $\mathrm{pHi}$

$\mathrm{pH}_{\mathrm{i}}$ of cells was assessed by flowcytometry using the pH-sensitive fluorescent probe BCECF-AM as described previously [24]. Cell suspensions in serum-free medium were washed and labeled with BCECF-AM. BCECF was successively excited at 490 and $440 \mathrm{~nm}$, and the resultant fluorescent signal was monitored at $535 \mathrm{~nm}$. Intensity ratio (490/440) was plotted vs. $\mathrm{pH}_{\mathrm{i}}$. In order to obtain the calibration curve, a linear regression within the $\mathrm{pH}_{\mathrm{i}}$ range 6.2-7.4 was obtained. To illustrate the decreased NHE1 activity in cariporide-treated and NHE1 silencing cells, the cells were acidified by an ammonium pulse and the rate of sodium-dependent $\mathrm{H}+$ extrusion was studied. Following exposure to $\mathrm{NH}_{4} \mathrm{Cl}, \mathrm{NH}_{3}$ enters the cells, binds $\mathrm{H}+$ to form $\mathrm{NH}_{4}+$ and thus leads to a transient cytosolic alkalinization. The subsequent removal of $\mathrm{NH}_{4} \mathrm{Cl}$ resulted in exit of $\mathrm{NH}_{3}$ leaving $\mathrm{H}+$ ions in the cell which causes cytosolic acidification.

Osteogenic and adipogenic differentiation of hUC-MSCS

Specialized cell culture media was purchased from Invitrogen and used according to the manufacturer's instruction to induce osteogenic and adipogenic differentiation of hUC-MSCs respectively in vitro. hUCMSCs were incubated in six-well plates in the presence or absence of $10 \mu \mathrm{M}$ cariporide for 21 days, and the medium was replaced every three days. The alkaline phosphatase (ALP) staining, Alizarin red (AR) staining and Von Kossa staining were used to detect osteogenic differentiation, and Oil Red 0 staining was used to detect adipogenic differentiation.

\section{Quantitative RT-PCR}

Total RNAs were isolated using Trizol reagent (Invitrogen, USA) according to the manufacturer's instruction. RNAs were reverse transcribed, and the relative mRNA expression levels of CEBPD, LPL, BMP, Osteocalcin, and $\beta$-Catenin were detected by Real-time PCR using ABI Prism 7500 sequence detection system (Applied Biosystems, USA). Meanwhile, GAPDH was used as internal reference gene. All primers are listed in Table 1. The Real-time PCR procedure included 3 min of pre-denaturation at $95{ }^{\circ} \mathrm{C}$, followed by 40 cycles of denaturation at $95^{\circ} \mathrm{C}$ for $15 \mathrm{~s}$ and $60{ }^{\circ} \mathrm{C}$ for $40 \mathrm{~s}$. Real-time PCR analysis of each sample was repeated in triplicate.

\section{Quantitative ALP assay}

Samples were lysed with $300 \mu \mathrm{L}$ of ALP lysis buffer and $0.5 \%$ Triton X-100 on 10th day during osteogenic differentiation. The hydrogel samples were homogenized with pipetting and sonicated for 10 min. BCA was conducted to assess the total protein to make sure that equal total protein was loaded in each group. The samples were vortexed and incubated with an alkaline buffer solution and a phosphatase substrate solution at $37^{\circ} \mathrm{C}$ for $15 \mathrm{~min}$. The ALP activity in $\mathrm{nmol} / \mathrm{min}$ was calculated by comparing samples' absorbance of p-nitrophenol product at $405 \mathrm{~nm}$ with that of p-nitrophenol standards.

\section{Western blot analysis}

The preparation of protein lysates and Western blot analysis were performed according to the manufacturer's instruction for each respective antibody. The primary antibodies used were anti- $\beta$-catenin (mouse monoclonal, Cell Signaling), and anti-GAPDH (rabbit monoclonal, Cell Signaling). 

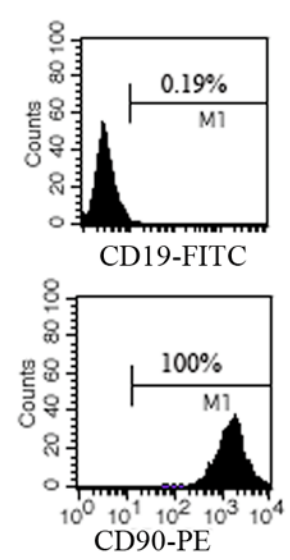
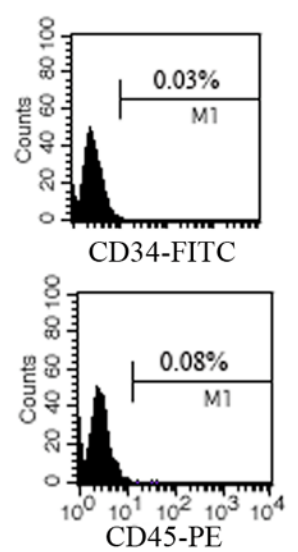
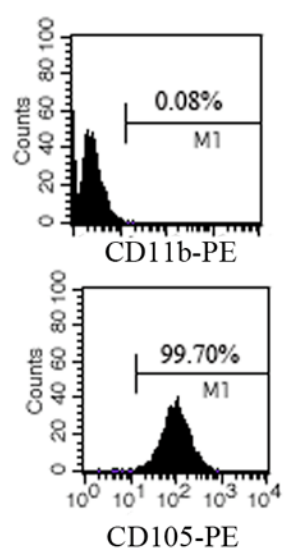
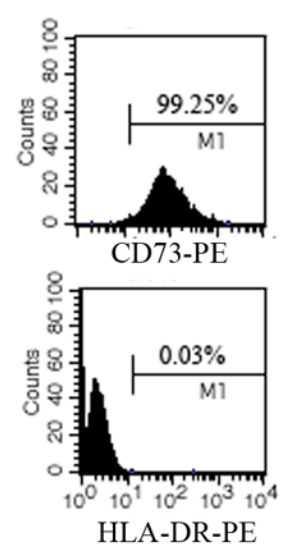

Fig. 1. Phenotype characteristics of hUC-MSCs. Surface markers of hUC-MSCs were detected by flowcytometric analysis. The experiment shown is representative of three performed.

\section{Statistical analysis}

The data was expressed as mean \pm SD over three independent experiments. Statistical significance of differences was analyzed by t-test for comparison between two groups. $P<0.05$ by two-tailed test was considered as statistically significant.

\section{Results}

\section{Characteristics of hUC-MSCs}

The surface phenotype was analyzed by flowcytometry according to the criteria defined by ISCT [10]. hUC-MSCs showed positive expression (>95\%) of CD73, CD90 and CD105, but negative $(<2 \%)$ for CD34, CD45, CD11b,CD19 and HLA-DR (Fig. 1). The cells possessed multipotent differentiation potential, as they could be induced to differentiate into osteoblasts and adipocytes under standard in vitro differential conditions.

\section{Cytotoxicity of cariporide on hUC-MSCS}

To explore the cytotoxicity of cariporide, hUC-MSCs were incubated with different concentrations of cariporide and MTT assay was performed. As Fig. 2a shows, cariporide inhibited hUC-MSCs growth at a concentration higher than $80 \mu \mathrm{M}$ but exerted little effect on hUC-MSCs at a low concentration. Finally, we chose a concentration of $10 \mu \mathrm{M}$ at the latter experiment to avoid the cytotoxicity of the inhibitor.

\section{Inhibition of NHE1 by cariporide decreased the pHi of hUC-MSCs}

To investigate the effect of NHE1 inhibitor on $\mathrm{pH}_{\mathrm{i}}$ of hUC-MSCs, $\mathrm{pH}_{\mathrm{i}}$ was measured in hUC-MSCs with treatment of $10 \mu \mathrm{M}$ cariporide for $24 \mathrm{~h}$ by using the fluorescent dye BCECFAM. As indicated in Fig. 2b, cariporide resulted in a decrease in $\mathrm{pH}_{\mathrm{i}}$ value of hUC-MSCs. Na+dependent alkalinization (Fig. 2c) showed that $\mathrm{pH}_{\mathrm{i}}$ recovered soon in the cells without any treatment, while $\mathrm{pH}_{\mathrm{i}}$ hardly recovered in cariporide-treated and NHE1 knock-down cells. This further confirmed that the decrease in $\mathrm{pH}_{\mathrm{i}}$ was due to the inhibition of NHE1 activity.

\section{NHE1 inhibition promoted the osteogenic differentiation of hUC-MSCS}

To explore the effect of NHE1 inhibition on the osteogenic differentiation of hUCMSCs, hUC-MSCs were incubated in the presence or absence of $10 \mu \mathrm{M}$ cariporide for 21 days. We performed the alkaline phosphatase (ALP) staining in the 10th day, Alizarin red (AR) staining and Von Kossa staining in the 21th day to detect osteogenic differentiation. As shown in Fig. 3a, the osteogenic differentiation of hUC-MSCs was up-regulated upon 
Fig. 2. Effect of cariporide on hUCMSCs cytotoxicity and $\mathrm{pH}_{\mathrm{i}}$ (a) Cytotoxicity of cariporide. hUC-MSCs were incubated with different concentrations of cariporide for $72 \mathrm{~h}$ and MTT was performed to measure the viability. Cariporide has little effect on hUC-MSCs viability at a low concentration. (b) Effect of cariporide on $\mathrm{pH}_{\mathrm{i}}$ of hUC-MSCs. hUC-MSCs were cultivated with $10 \mu \mathrm{M}$ cariporide for 24 h. $\mathrm{pH}_{\mathrm{i}}$ was measured by using the fluorescent dye BCECF-AM ${ }^{*} P$ $<0.05$ ). (c) Na+-dependent alkalinization. In the presence of $\mathrm{Na}+$, $\mathrm{pH}_{\mathrm{i}}$ recovered soon in the cells without any treatment, while the $\mathrm{pH}_{\mathrm{i}}$ hardly recovered in the cariporide-treatment cells and NHE1 silencing cells.

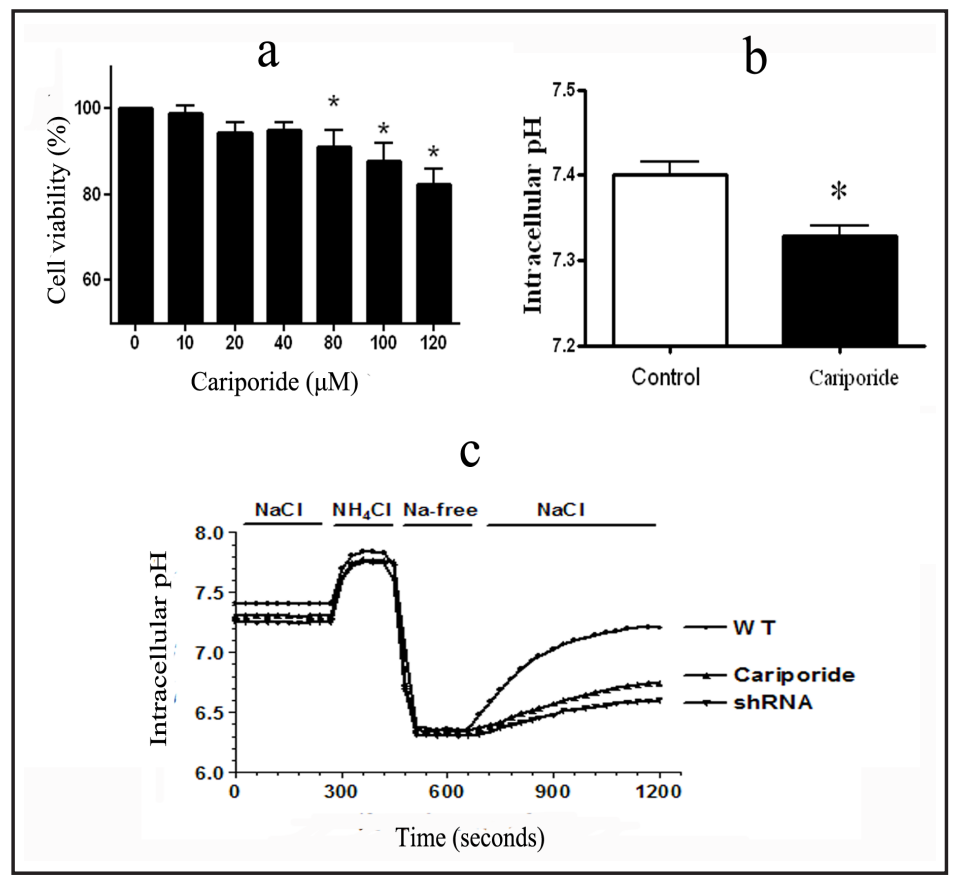

Fig. 3. Effect of NHE1 inhibition on hUC-MSCs osteogenic differentiation. (a) hUC-MSCs were incubated in osteogenic differential culture media in the presence or absence of $10 \mu \mathrm{M}$ cariporide for 21 days. The alkaline phosphatase (ALP) staining in the 10th day, Alizarin red (AR) staining and Von Kossa staining in the 21th day were used to characterize osteogenic differentiation. (b) hUCMSCs were incubated in osteogenic differential culture media in the pre-

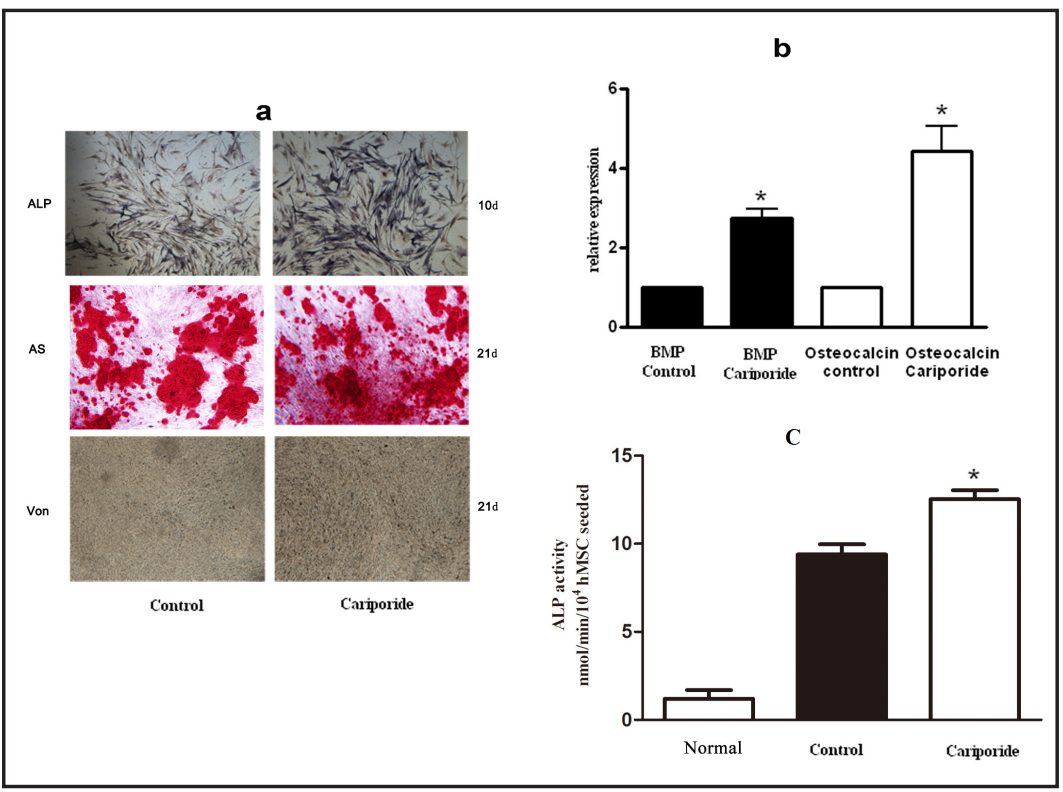
sence or absence of $10 \mu \mathrm{M}$ cariporide for 14 days. Total RNA was collected and quantitative RT-PCR was performed to assess the expression of the indicated genes $\left({ }^{*} P<0.05\right)$. (c) The ALP activity was measured by quantitative ALP assay on 10 th day during osteogenic differentiation $\left({ }^{*} P<0.05\right)$.

cariporide treatment. Quantitative RT-PCR analysis (Fig. 3b) showed that the expression of main osteoblast markers BMP and Osteocalcin were also up-regulated upon cariporide treatment for 14 days. To further confirm the effect, quantitative ALP assay was performed. As shown in Fig. 3c, the ALP activity increased significantly in cariporide treatment group compared with control group. These results indicated that NHE1 inhibition promoted the osteogenic differentiation of hUC-MSCs. 
Fig. 4. NHE1 ShRNA knock-down experiment. (a) Effect of NHE1 shRNA knock-down on $\mathrm{pH}_{\mathrm{i}}$ of hUC-MSCs $\left({ }^{*} \mathrm{P}<0.05\right)$. (b) The alkaline phosphatase (ALP) staining in the 10th day was used to detected osteogenic differentiation. (c) The ALP activity was measured by quantitative ALP assay on 10th day during osteogenic differentiation. The normal group is a negative control, measuring the ALP activity of undifferentiated hUCMSCs cultured in complete culture medium $\left({ }^{*} \mathrm{P}<0.05\right)$.

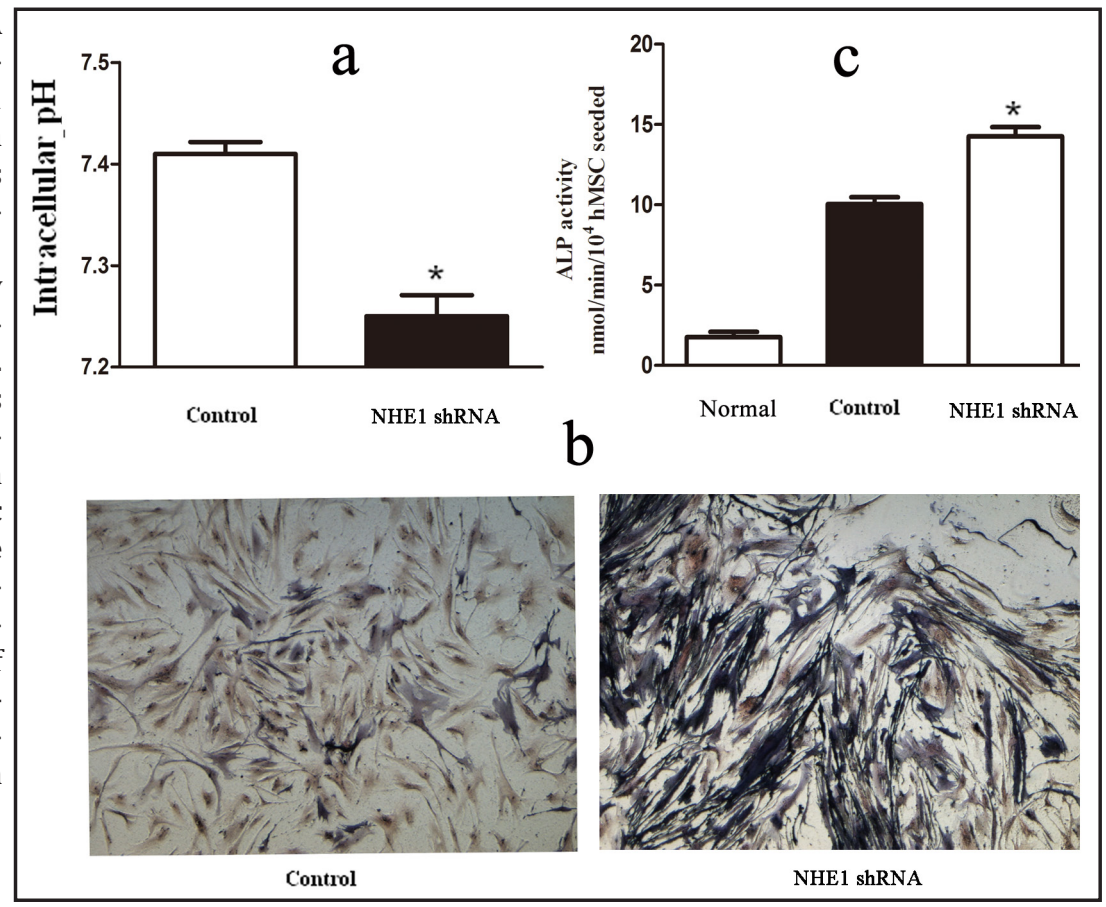

Fig. 5. Effect of NHE1 inhibition on hUC-MSCs adipogenic differentiation. (a) hUC-MSCs were incubated in adipogenic differential culture media in the presence or absence of $10 \mu \mathrm{M}$ cariporide for 21 days. Oil Red 0 staining was used to characterize adipogenic differentiation. (b) hUC-MSCs were incubated in adipogenic differential culture media in the presence or absence of $10 \mu \mathrm{M}$ cariporide for 21days. Total RNA was collected and quantitative RT-PCR was performed to assess the expression of the indicated genes.

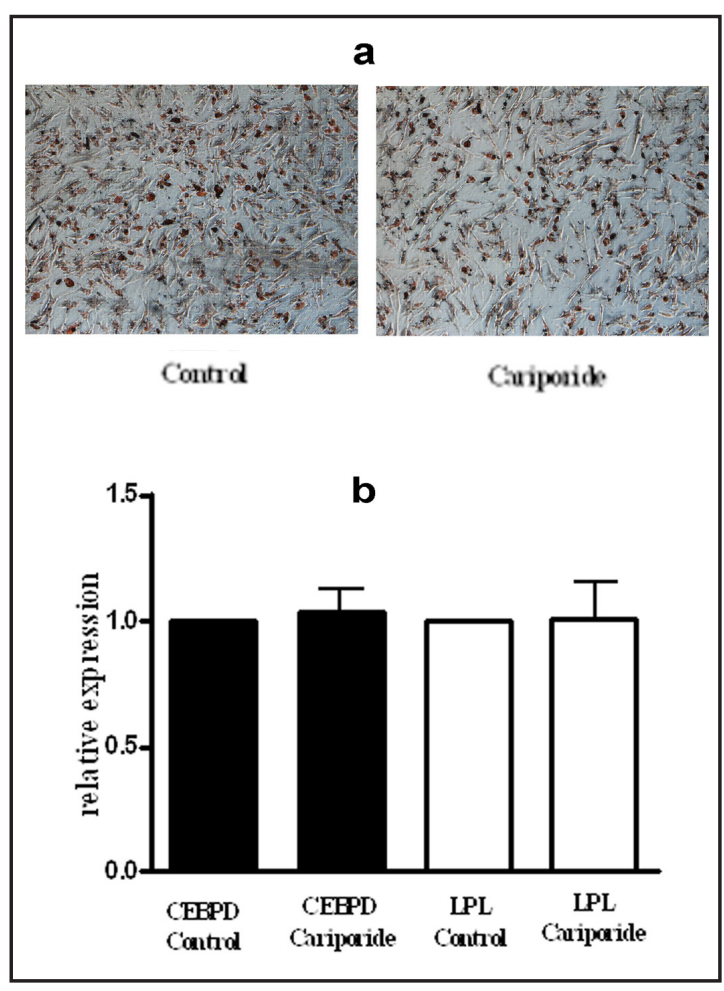

NHE1 knock-down experiment

To further confirm the effect of NHE1 inhibition on osteogenic differentiation, we performed the NHE1 knock-down experiment. As shown in Fig. 4, NHE1 knock-down induced a decrease in $\mathrm{pH}_{\mathrm{i}}$ and promoted the osteogenic differentiation of hUC-MSCs.

Effect of NHE1 inhibition on adipogenic differentiation of hUC-MSCS

To evaluate the effect of NHE1 inhibition on adipogenic differentiation of hUC-MSCs, hUC-MSCs were incubated in the presence or absence of $10 \mu \mathrm{M}$ cariporide for 21 days, Oil Red 
Fig. 6. Effect of NHE1 inhibition on expression of $\beta$-catenin in osteogenic differentiation of hUC-MSCs. (a) hUC-MSCs were incubated in osteogenic differential culture media in the presence or absence of $10 \mu \mathrm{M}$ cariporide for 3 days. Total RNA was collected and quantitative RT-PCR was performed to assess the expression of $\beta$-catenin in mRNA level $\left({ }^{*} P<0.05\right)$. (b) hUC-MSCs were incubated in

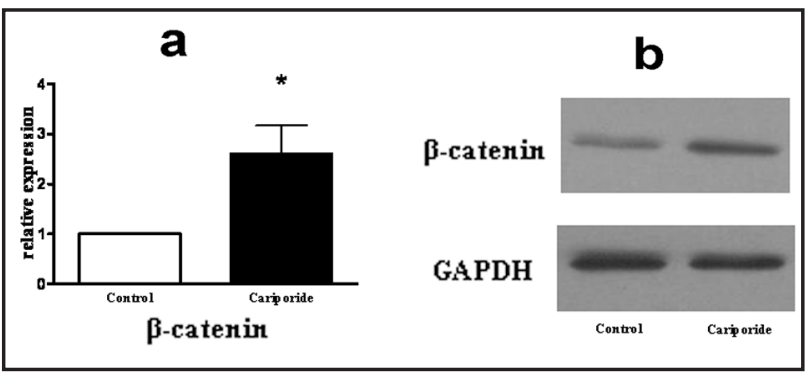
osteogenic differential culture media in the presence or absence of $10 \mu \mathrm{M}$ cariporide for 3 days. Western blot was performed to assess the expression of $\beta$-catenin in protein level.

O staining was used to detect adipogenic differentiation. As shown in Fig. 5a, the adipogenic differentiation of hUC-MSCs was not significantly affected upon cariporide treatment. Quantitative RT-PCR analysis (Fig. 5b) showed that the expression of main adipogenic markers CEBPD and LPL were also not significantly affected upon cariporide treatment. These results indicated that NHE1 inhibition had little effect on adipogenic differentiation of hUC-MSCs.

NHE1 inhibition up-regulated the expression of $\beta$-catenin in osteogenic differentiation of hUC-MSCS

We then investigated the potential mechanisms through which NHE1 inhibition promoted the osteogenic differentiation of hUC-MSCs. Wnt/ $\beta$-catenin signaling pathway has been proven to play an important role in osteoblastogenesis. Quantitative RT-PCR (Fig. 6a) and Western blot (Fig. 6b) analysis showed that the expression of $\beta$-catenin was upregulated upon cariporide treatment for 3 days.

\section{Discussion}

It is well known that $\mathrm{pH}_{\mathrm{i}}$ is important for cell metabolism since many cellular mechanisms are sensitive to changes in $\mathrm{pH}_{\mathrm{i}}$ [25-28]. In normal differentiated adult cells, $\mathrm{pH}_{\mathrm{i}}$ is generally $\sim 7.2$. The cancer cells and the stem cells, however, possess a higher $\mathrm{pH}_{\mathrm{i}}[1]$. The $\mathrm{pH}_{\mathrm{i}}$ is regulated by transport systems including proton extruders like NHE, the $\mathrm{H}+$ pump, and/ or bicarbonate transporters which mediate the influx or extrusion of bicarbonate [29]. One of the most important transporters is NHE1, which is a ubiquitously expressed member of the $\mathrm{Na}+\mathrm{H}+$ exchanger family. To confirm the effect of $\mathrm{NHE} 1$ on $\mathrm{pH}_{\mathrm{i}}$, we performed the $\mathrm{Na}+-$ dependent alkalinization assay. The slight recovery of $\mathrm{pH}_{\mathrm{i}}$ in cariporide-treated and NHE1 knock-down cells indicated that other $\mathrm{pH}_{\mathrm{i}}$ regulatory factors may be involved in this process. For instance, monocarboxylate transporters (MCTs) [30,31] and the plasma membrane proton pump vacuolar ATPase (V-ATPase) are also responsible for the regulation of $\mathrm{pH}_{\mathrm{i}}$ in $\mathrm{Na}$ +-independent manner [32-34]. Our previous study has shown that targeted inhibition of NHE1 induced differentiation of K562 leukemia cells and NHE1 was highly expressed in mesenchymal stem cell. So, we presume that whether decreased $\mathrm{pH}_{\mathrm{i}}$ could also induce differentiation of mesenchymal stem cells.

Mesenchymal stem cells (MSCs), also known as mesenchymal stromal stem cells and mesenchymal stromal cells, are a heterogeneous multipotent population of stromal cells with fibroblast-like morphology and proliferate in vitro as plastic-adherent cells. They also possess the properties to form colonies in vitro and differentiate into osteoblasts, chondrocytes and adipocytes. Human umbilical cord-derived mesenchymal stem cells (hUCMSCs), have incomparable advantages: (1) Take full advantage of discarded materials from the new born baby, which are easily accessible and less troublesome in ethical controversy. (2) hUC-MSCs are readily long-time preparation and can be scaled up in large numbers 
because of short doubling time. (3) hUC-MSCs contain both human embryonic stem cells and human mesenchymal stem cells markers. (4) hUC-MSCs maintain "stemness" for several serial passages. hUC-MSCs offer tremendous promise in cellular therapeutics [35].

In this experiment, the surface phenotype and functional characteristics of hUC-MSCs which are obtained from human umbilical cord were analyzed. To explore the effect of NHE1 inhibition on differentiation of MSCs, selective NHE1 inhibitor cariporide was used to treat hUC-MSCs in different differential culture media. To avoid the cytotoxicity, we selected a low concentration of $10 \mu \mathrm{M}$. In this concentration, the growth of hUC-MSCs was not affected but the $\mathrm{pH}_{\mathrm{i}}$ was decreased significantly.

The differentiation of hUC-MSCs was confirmed by both cytochemical staining and differential marker expression. The alkaline phosphatase staining in the 10th day, Alizarin red staining and Von Kossa staining in the 21th day showed that osteogenic differentiation of hUC-MSCs was up-regulated upon cariporide treatment. The up-regulation of the main osteoblast markers BMP and Osteocalcin expression and quantitative ALP assay confirmed the effect. However, the Oil Red 0 staining and main adipogenic markers CEBPD and LPL expression showed that the adipogenic differentiation was not affected upon cariporide treatment. NHE1 inhibition differentially contributes to hUC-MSCs differentiation. The differential effect may have potential clinical value, especially osteoblast associated disease, such as tissue engineering, bone fracture healing and bone disease in patients with multiple myeloma (MM) [36, 37]. Our recent results have shown the similar effect on BM-MSCs derived from patients with multiple myeloma. Why adipogenic differentiation was not affected upon cariporide treatment? In our opinions, the hUC-MSCs are prone to differentiated into adipocyte so easily in ex vivo culture media that the effect of cariporide is negligible. However, the effect in vivo needs further investigations.

Lots of evidence indicated that Wnt/ $\beta$-catenin signaling pathway plays an important role in osteoblastogenesis [38-40]. Our results showed that cariporide induced MSCs differentiation towards osteoblast, so we explored whether Wnt/ $\beta$-catenin signaling is involved in this process. Indeed, cariporide treatment up-regulated $\beta$-catenin expression in both mRNA level and protein level suggesting that NHE1 inhibition up-regulated the osteogenic differentiation of hUC-MSCs at least in part through the up-regulation of $\beta$-catenin. NHE1 inhibition may up-regulate the expression of $\beta$-catenin through MAPK signaling pathway. Previous study has shown that NHE1 plays a central role in the regulation of MAPK activity. Takewaki et al. found that the specific NHE1 inhibitor cariporide partially inhibited stretch-induced activation of ERK as well as of NF- $\mathrm{KB}$ in cultured cardiomyocytes [41]. Mukhin et al. showed that Ras and MEK1/2 activation by Ang II or ET-1 were also attenuated by NHE1 inhibition, indicating that the effect of NHE1 was at or above the Ras level [42]. Chen et al. demonstrated that HOE642 prevented glucose-induced ERK1/2 activation and hypertrophy in a high glucose model of cardiomyocyte hypertrophy, further supporting the notion that NHE1-ERK1/2 pathway plays an important role in hypertrophy development [43]. MAPK is involved in $\beta$-catenin expression [44, 45]. But how NHE1 inhibition upregulated the expression of $\beta$-catenin and which other signaling pathway is also involved needs further study.

In summary, our study demonstrates for the first time that decreased intracellular $\mathrm{pH}$ induced by cariporide differentially contributed to human umbilical cord-derived mesenchymal stem cells differentiation. These findings may open a new way for better use of MSCs in clinical trials.

\section{Acknowledgements}

This work was supported by a grant from the National Natural Science General Program Foundation of China (No.81170510) and National Natural Science Major Program Foundation of China (No.81090410). Disclosure: The authors declare no competing financial interests. 
Gao et al.: Decreased $\mathrm{pH}_{\mathrm{i}}$ and MSCs Differentiation

\section{References}

1 Webb BA, Chimenti M, Jacobson MP, Barber DL: Dysregulated ph: A perfect storm for cancer progression. Nat Rev Cancer 2011;11:671-677.

-2 Rich IN, Worthington-White D, Garden OA, Musk P: Apoptosis of leukemic cells accompanies reduction in intracellular $\mathrm{pH}$ after targeted inhibition of the $\mathrm{Na}^{+} / \mathrm{H}^{+}$exchanger. Blood 2000;95:1427-1434.

- Slepkov ER, Rainey JK, Sykes BD, Fliegel L: Structural and functional analysis of the $\mathrm{Na}^{+} / \mathrm{H}^{+}$exchanger. Biochem J 2007;401:623-633.

4 Malo ME, Fliegel L: Physiological role and regulation of the $\mathrm{Na}^{+} / \mathrm{H}^{+}$exchanger. Can J Physiol Pharmacol 2006;84:1081-1095.

5 Counillon L, Pouyssegur J: The expanding family of eucaryotic $\mathrm{Na}^{+} / \mathrm{H}^{+}$exchangers. J Biol Chem 2000;275:14.

6 Wakabayashi S, Shigekawa M, Pouyssegur J: Molecular physiology of vertebrate $\mathrm{Na}^{+} / \mathrm{H}^{+}$exchangers. Physiol Rev 1997;77:51-74.

7 Reshkin SJ, Cardone RA, Harguindey S: $\mathrm{Na}^{+} / \mathrm{H}^{+}$exchanger, $\mathrm{pH}$ regulation and cancer. Recent Pat Anti-cancer Drug Discov 2013;8:85-99.

8 Jin W, Li Q, Wang J, Chang G, Lin Y, Li H, Wang L, Gao W, Pang T: $\mathrm{Na}^{+} / \mathrm{H}^{+}$exchanger 1 inhibition contributes to K562 leukaemic cell differentiation. Cell Biol Int 2012;36:739-745.

- Horwitz EM, Le Blanc K, Dominici M, Mueller I, Slaper-Cortenbach I, Marini FC, Deans RJ, Krause DS, Keating A: Clarification of the nomenclature for MSC: The international society for cellular therapy position statement. Cytotherapy 2005;7:393-395.

10 Dominici M, Le Blanc K, Mueller I, Slaper-Cortenbach I, Marini F, Krause D, Deans R, Keating A, Prockop D, Horwitz E: Minimal criteria for defining multipotent mesenchymal stromal cells. The international society for cellular therapy position statement. Cytotherapy 2006;8:315-317.

11 Pittenger MF, Mackay AM, Beck SC, Jaiswal RK, Douglas R, Mosca JD, Moorman MA, Simonetti DW, Craig S, Marshak DR: Multilineage potential of adult human mesenchymal stem cells. Science 1999;284:143-147.

12 Prockop DJ: Marrow stromal cells as stem cells for nonhematopoietic tissues. Science 1997;276:71-74.

13 De Ugarte DA, Alfonso Z, Zuk PA, Elbarbary A, Zhu M, Ashjian P, Benhaim P, Hedrick MH, Fraser JK: Differential expression of stem cell mobilization-associated molecules on multi-lineage cells from adipose tissue and bone marrow. Immunol Lett 2003;89:267-270.

14 Hong SH, Gang EJ, Jeong JA, Ahn C, Hwang SH, Yang IH, Park HK, Han H, Kim H: In vitro differentiation of human umbilical cord blood-derived mesenchymal stem cells into hepatocyte-like cells. Biochem Biophys Res Commun 2005;330:1153-1161.

15 Covas DT, Siufi JL, Silva AR, Orellana MD: Isolation and culture of umbilical vein mesenchymal stem cells. Braz J Med Biol Res 2003;36:1179-1183.

16 Mihu CM, Rus Ciuca D, Soritau O, Susman S, Mihu D: Isolation and characterization of mesenchymal stem cells from the amniotic membrane. Rom J Morphol Embryol 2009;50:73-77.

17 Zhang Q, Shi S, Liu Y, Uyanne J, Shi Y, Le AD: Mesenchymal stem cells derived from human gingiva are capable of immunomodulatory functions and ameliorate inflammation-related tissue destruction in experimental colitis. J Immunol 2009;183:7787-7798.

18 Siepe M, Thomsen AR, Duerkopp N, Krause U, Forster K, Hezel P, Beyersdorf F, Schlensak C, Sudkamp NP, Bosse R, Niemeyer P: Human neonatal thymus-derived mesenchymal stromal cells: Characterization, differentiation, and immunomodulatory properties. Tissue Eng Part A 2009;15:1787-1796.

19 Miao Z, Jin J, Chen L, Zhu J, Huang W, Zhao J, Qian H, Zhang X: Isolation of mesenchymal stem cells from human placenta: Comparison with human bone marrow mesenchymal stem cells. Cell Biol Int 2006;30:681-687.

-20 Sakaguchi Y, Sekiya I, Yagishita K, Muneta T: Comparison of human stem cells derived from various mesenchymal tissues: Superiority of synovium as a cell source. Arthritis Rheum 2005;52:2521-2529.

21 Campagnoli C, Roberts IA, Kumar S, Bennett PR, Bellantuono I, Fisk NM: Identification of mesenchymal stem/progenitor cells in human first-trimester fetal blood, liver, and bone marrow. Blood 2001;98:23962402.

22 Scholz W, Albus U, Counillon L, Gogelein H, Lang HJ, Linz W, Weichert A, Scholkens BA: Protective effects of HOE642, a selective sodium-hydrogen exchange subtype 1 inhibitor, on cardiac ischaemia and reperfusion. Cardiovasc Res 1995;29:260-268. 
Gao et al.: Decreased $\mathrm{pH}_{\mathrm{i}}$ and MSCs Differentiation

23 Aguilar S, Nye E, Chan J, Loebinger M, Spencer-Dene B, Fisk N, Stamp G, Bonnet D, Janes SM: Murine but not human mesenchymal stem cells generate osteosarcoma-like lesions in the lung. Stem Cells 2007;25:15861594.

24 Gao W, Chang G, Wang J, Jin W, Wang L, Lin Y, Li H, Ma L, Li Q, Pang T: Inhibition of K562 leukemia angiogenesis and growth by selective $\mathrm{Na}^{+} / \mathrm{H}^{+}$exchanger inhibitor cariporide through down-regulation of pro-angiogenesis factor VEGF. Leuk Res 2011;35:1506-1511.

-25 Semina OV, Semenets TN, Zamulaeva IA, Selivanova EI, Iljina TP, Maliutina YV, Semin DY, Deigin VI, Saenko AS: Dipeptide gamma-d-glu-d-trp (thymodepressin) inhibits migration of CD34+ cells from the bone marrow into peripheral blood during tumor growth. Bull Exp Biol Med 2008;146:96-99.

26 Grinstein S, Woodside M, Sardet C, Pouyssegur J, Rotin D: Activation of the $\mathrm{Na}^{+} / \mathrm{H}^{+}$antiporter during cell volume regulation. Evidence for a phosphorylation-independent mechanism. J Biol Chem 1992;267:2382323828.

27 Grinstein S, Rotin D, Mason $\mathrm{MJ}$ : $\mathrm{Na}^{+} / \mathrm{H}^{+}$exchange and growth factor-induced cytosolic $\mathrm{pH}$ changes. Role in cellular proliferation. Biochim Biophys Acta 1989;988:73-97.

28 Lu Y, Pang T, Wang J, Xiong D, Ma L, Li B, Li Q, Wakabayashi S: Down-regulation of p-glycoprotein expression by sustained intracellular acidification in K562/DOX cells. Biochem Biophys Res Commun 2008;377:441-446.

29 Puceat M: pHi regulatory ion transporters: An update on structure, regulation and cell function. Cell Mol Life Sci 1999;55:1216-1229.

-30 Enerson BE, Drewes LR: Molecular features, regulation, and function of monocarboxylate transporters: Implications for drug delivery. J Pharm Sci 2003;92:1531-1544.

-31 Halestrap AP, Price NT: The proton-linked monocarboxylate transporter (MCT) family: structure, function and regulation. Biochem J 1999;343:281-299.

32 Martinez-Zaguilan R, Lynch RM, Martinez GM, Gillies RJ: Vacuolar-type H+-ATPases are functionally expressed in plasma membranes of human tumor cells. Am J Physiol 1993;265:C1015-1029.

33 Nishi T, Forgac M: The vacuolar H+-ATPases--nature's most versatile proton pumps. Nat Rev Mol Cell Biol 2002;3:94-103.

34 Perez-Sayans M, Somoza-Martin JM, Barros-Angueira F, Rey JM, Garcia-Garcia A: V-ATPase inhibitors and implication in cancer treatment. Cancer Treat Rev 2009;35:707-713.

-35 Fan CG, Zhang QJ, Zhou JR: Therapeutic potentials of mesenchymal stem cells derived from human umbilical cord. Stem Cell Rev 2011;7:195-207.

-36 Schneider RK, Puellen A, Kramann R, Raupach K, Bornemann J, Knuechel R, Perez-Bouza A, Neuss S: The osteogenic differentiation of adult bone marrow and perinatal umbilical mesenchymal stem cells and matrix remodelling in three-dimensional collagen scaffolds. Biomaterials 2010;31:467-480.

37 Roodman GD: Novel targets for myeloma bone disease. Expert Opin Ther Targets 2008;12:1377-1387.

-38 Tian E, Zhan F, Walker R, Rasmussen E, Ma Y, Barlogie B, Shaughnessy JD Jr: The role of the wntsignaling antagonist dkk1 in the development of osteolytic lesions in multiple myeloma. $\mathrm{N}$ Engl J Med 2003;349:2483-2494.

-39 Westendorf JJ, Kahler RA, Schroeder TM: Wnt signaling in osteoblasts and bone diseases. Gene 2004;341:19-39.

-40 Gaur T, Lengner CJ, Hovhannisyan H, Bhat RA, Bodine PV, Komm BS, Javed A, van Wijnen AJ, Stein JL, Stein GS, Lian JB: Canonical wnt signaling promotes osteogenesis by directly stimulating runx2 gene expression. J Biol Chem 2005;280:33132-33140.

-41 Takewaki S, Kuro-o M, Hiroi Y, Yamazaki T, Noguchi T, Miyagishi A, Nakahara K, Aikawa M, Manabe I, Yazaki Y, et al.: Activation of $\mathrm{Na}+\mathrm{H}+$ antiporter (NHE-1) gene expression during growth, hypertrophy and proliferation of the rabbit cardiovascular system. J Mol Cell Cardiol 1995;27:729-742.

42 Mukhin YV, Garnovskaya MN, Ullian ME, Raymond JR: ERK is regulated by sodium-proton exchanger in rat aortic vascular smooth muscle cells. J Biol Chem 2004;279:1845-1852.

43 Chen S, Khan ZA, Karmazyn M, Chakrabarti S: Role of endothelin-1, sodium hydrogen exchanger-1 and mitogen activated protein kinase (MAPK) activation in glucose-induced cardiomyocyte hypertrophy. Diabetes Metab Res Rev 2007;23:356-367.

44 Bikkavilli RK, Feigin ME, Malbon CC: p38 mitogen-activated protein kinase regulates canonical wnt- $\beta$ catenin signaling by inactivation of GSK3 $\beta$. J Cell Sci 2008;121:3598-3607.

$\checkmark 45$ Caraci F, Gili E, Calafiore M, Failla M, La Rosa C, Crimi N, Sortino MA, Nicoletti F, Copani A, Vancheri C: TGF- $\beta 1$ targets the GSK-3 $\beta / \beta$-catenin pathway via ERK activation in the transition of human lung fibroblasts into myofibroblasts. Pharmacol Res 2008;57:274-282. 